MODELING, IDENTIFICATION AND CONTROL, 1981, VOL. 2, NO. 2, 95-106

doi: $10.4173 /$ mic.1981.24

\title{
Pressure transients and control in liquid propane caverns $\dagger$
}

\author{
O. A. ASBJØRNSEN $\ddagger$ P. H. AAS§ and J. HANCKE $\ddagger$
}

Keywords: process modelling, process simulation, parameter estimation, liquid propane caverns.

This paper shows how a very simple model of the process dynamics of a LPG cavern may be developed, and demonstrates the applicability of this model. The model is used to simulate the consequences of malfunctioning control equipment during loading of the cavern, to design a simple feed forward control strategy for the feed temperature and to estimate the model parameters from real plant experiments. From these experiments the heat transfer from the vapor phase to the rock and the heat conductivity of the rock itself are estimated. The parameters so obtained are plausible and may be used for further simulations or design and evaluation of similar caverns. The results of the model fitting show that the material balance is accurate within less than $1 \%$ while the energy balance is accurate to withon $0.07^{\circ} \mathrm{C}$ or about $3 \%$ of the peak value of the temperature transient during loading.

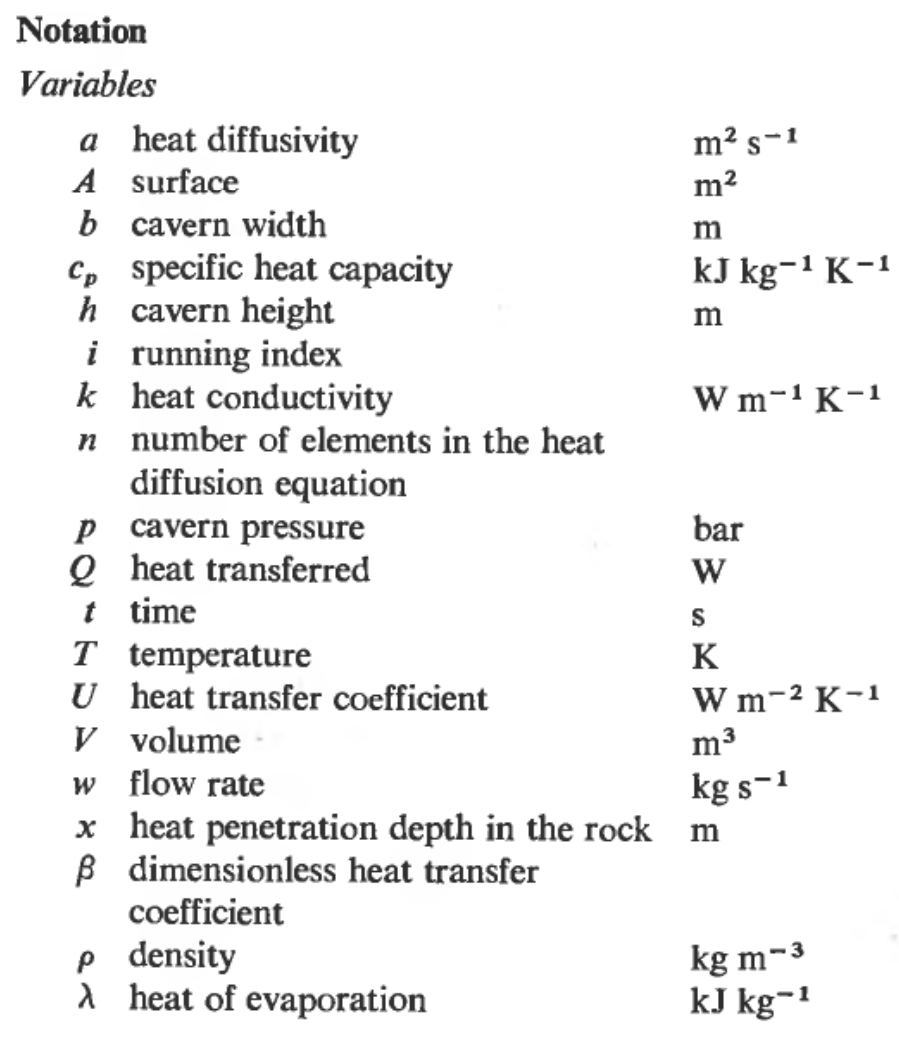

Received 22 October 1979. 1979.

† Paper presented at the 72nd AIChE Annual Meeting, San Francisco, 25-29 November

$\ddagger$ Norsk Hydro a.s., Porsgrunn Fabrikker, Postboks 110, N-3901 Porsgrunn, Norway.

$\S$ Norsk Hydro a.s., Engineering Division, Lørenfaret 3, Oslo 5, Norway. 

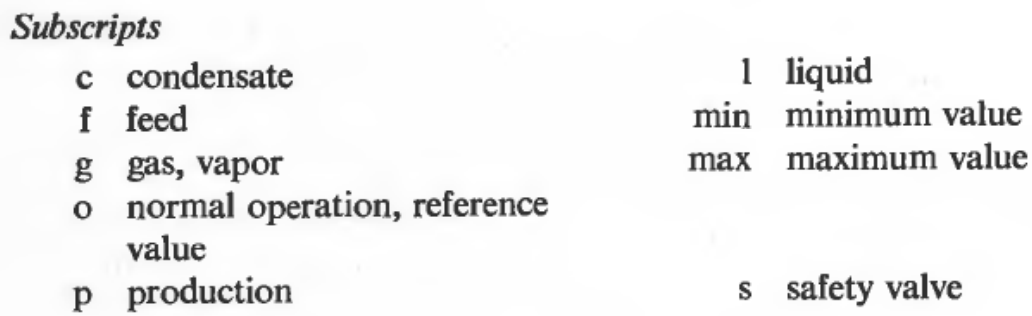

\section{Introduction}

Modern rock excavation technique has developed to an efficiency and a standard which make the excavation of large underground caverns economically feasible and attractive alternatives to pressurized storages of liquified light hydrocarbons. The technique requires a good quality rock which should preferably not need any surface treatment after the excavation. When light hydrocarbons, like, for example, propane are stored in artificial caverns of this type, the temperature conditions in the rock lead to a gauge pressure which would correspond to the vapor pressure at the cavern temperature. This pressure should be balanced by the ground water pressure in the cavern, such that ground water leaks into the cavern. Under no circumstances should hydrocarbon liquid or vapor leak out from the storage through the pores or the cracks of the rock. This requires a certain depth of the cavern where the temperature is low enough and the ground water pressure is high enough to more than balance the vapor pressure of the cavern under all circumstances. Such depths would typically be around $100 \mathrm{~m}$.

When the cavern is sealed off and the storage is taken into operation, a sealed-off vapor volume above the liquid phase will compress and expand when liquid is loaded into or unloaded out from the cavern respectively. This generates pressure and temperature transients in the cavern, and these transients should be kept under control within fairly narrow limits. If the pressure in the cavern reaches a value which is close to the ground water pressure at the cavern location, one is in danger of losing the ground water in the vicinity of the cavern. This may cause hazardous conditions and should be avoided under all circumstances. Hence, it is important to be able to evaluate the cavern dynamics and the nature of the pressure and temperature transients. The purpose of this paper is to demonstrate the practical applicability of a very simple mathematical model and how the parameters in this model may be estimated from real plant behaviour. The application of the model is fourfold. First, to simulate the possible consequences of malfunctioning control equipment during the loading of the cavern. Secondly, to devise a simple control strategy for the loading of the cavern, which is the most risky operation. Thirdly, to get a better physical and theoretical insight into the dynamic behaviour of a process storage unit of this size and nature, typically around $10^{5} \mathrm{~m}^{3}$ about $100 \mathrm{~m}$ below sea level in an arbitrary rock quality. Fourthly, to examine if a mathematical model of this nature could possibly improve the metering accuracy of the loading and unloading of the cavern.

\section{A simple mathematical model of the cavern}

If one ignores the size of the cavern, the typical nature of a one component twophase system in a sealed-off volume with liquid feed and take off, is indeed very trivial. In principle, it may be treated as, for example, a vapor-liquid thermometer found 
in any textbook on process dynamics (Luyben 1973, Douglas 1972, Harnot 1964). The question is, however, if such a simple model may apply to a sealed-off volume of these enormous dimensions. The answer is yes, and the paper shows how well the application really is for a cavern of $125000 \mathrm{~m}^{3}$.

A typical underground cavern is illustrated in Fig. 1. This shows the feed system, the take-off system, the ground water drainage and the safety valve arrangement. At about $100 \mathrm{~m}$ depth, the rock temperature is fairly constant throughout the year at a level which depends upon the latitude. At about $60^{\circ} \mathrm{N}$, the temperature is around $6-7^{\circ} \mathrm{C}$.

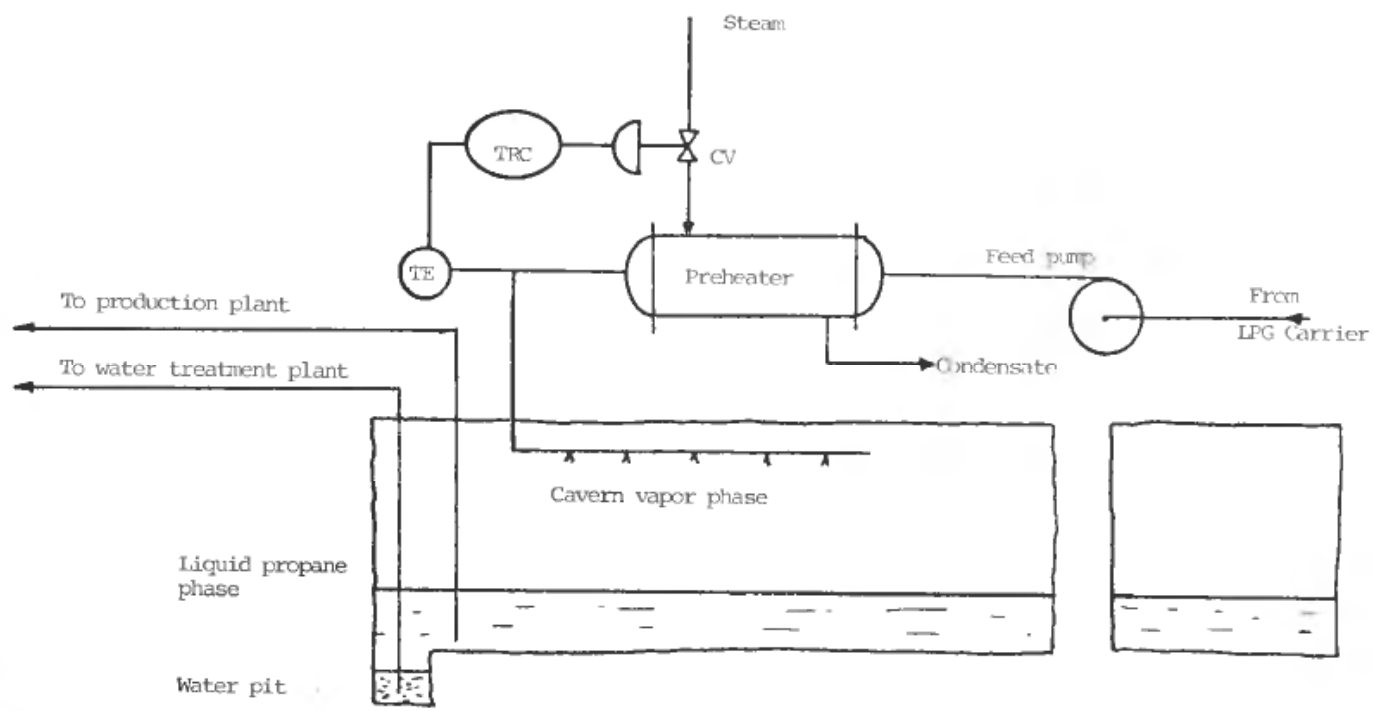

Figure 1. Schematic flowsheet of a liquid propane cavern.

The temperature of the feed to the cavern should normally be below the storage temperature, to facilitate vapor condensation during compression as the liquid level rises. On the other hand, the feed temperature should not be so low that hydrates are being formed in the hydrocarbons in contact with the ground water. Hence, there is an important control loop for the feed preheater as shown in Fig. 1.

The ground water is collected in a pit and pumped out to a water treatment plant. The ground water drainage is controlled by the water level in the pit. The cavern has a safety-valve arrangement for emergency and a take-off line to the production plant. The safety valve opens automatically at a certain pressure in the cavern and the takeoff line to the production plant normally has a flow control.

The feed may be introduced as a liquid spray at the top of the cavern, the idea being that the feed should have an extremely good contact with the vapor to ensure equilibrium between the vapor phase and the feed. The feed may also be introduced into the bottom of the cavern below the liquid surface, the idea again being that advantage should be taken of the enormous liquid time constant for damping out temperature and hence pressure transients. These two situations will be the basis for two extreme mathematical models, one segregated single-phase vapor model in 
equilibrium with the liquid feed spray, and one combined two-phase model where the complete liquid phase and the vapor phase in the cavern are both perfectly mixed and in equilibrium.

\section{A single-phase vapor model}

If only the vapor phase is taken into account and the feed is considered to be sprayed into the vapor volume and in thermodynamic equilibrium, the transient material balance of the vapor phase may be written in terms of the feed, the flow through the safety valve and the liquid flow leaving the vapor phase into the liquid phase;

$$
\frac{d}{d t}\left(V_{\mathrm{g}} \rho_{\mathrm{g}}\right)=w_{\mathrm{f}}-w_{\mathrm{c}}-w_{\mathrm{s}}
$$

The model for the flow rate through the safety valve is taken to be a linear function of pressure in the cavern;

$$
\left.\begin{array}{l}
w_{\mathrm{s}}=0 \quad \text { if } \quad p \leqslant p_{\min } \\
w_{\mathrm{s}}=w_{\mathrm{o}} \quad \text { if } \quad p \geqslant p_{\max } \\
w_{\mathrm{s}}=\left(p-p_{\min }\right) w_{\mathrm{o}} /\left(p_{\max }-p_{\min }\right) \text { otherwise }
\end{array}\right\}
$$

The vapor phase will be compressed by the rising liquid level during loading of the cavern. The effect of this volume compression by the liquid interface may be written in terms of liquid entering the liquid phase and product taken off,

$$
\frac{d}{d t}\left(V_{\mathrm{s}}\right)=-\left(w_{\mathrm{c}}-w_{\mathrm{p}}\right) / \rho_{1}
$$

Since there is a one to one correlation between pressure and density or temperature and density of pure saturated propane vapor, the material balance may be written in terms of pressure or temperature,

$$
V_{\mathrm{g}} \frac{d \rho_{\mathrm{g}}}{d T} \dot{T}=w_{\mathrm{f}}-\left(1-\rho_{\mathrm{g}} / \rho_{1}\right) w_{\mathrm{c}}-\left(\rho_{\mathrm{g}} / \rho_{1}\right) w_{\mathrm{p}}-w_{\mathrm{s}}
$$

The energy balance of the vapor phase alone may be written in terms of the ethalpy of the feed, the ethalpy of condensing vapor or evaporating feed and the energy loss into the vapor phase surrounding. It should be emphasized that this model ignores the energy state of the liquid in the cavern, since the latent heat of evaporation and condensation is taken from or released to the vapor phase alone

$$
\frac{d}{d t}\left(V_{\mathrm{g}} \rho_{\mathrm{g}} c_{\mathrm{pg}} T-V_{\mathrm{g}} p\right)=w_{\mathrm{f}} c_{p 1} T_{\mathrm{f}}+\frac{p}{\rho_{1}}\left(w_{\mathrm{c}}-w_{\mathrm{p}}\right)-w_{\mathrm{c}} c_{p 1} T-w_{\mathrm{s}}\left(\lambda+c_{p 1} T\right)-Q
$$

giving

$$
V_{\mathrm{g}}\left(\rho_{\mathrm{g}} c_{p \mathrm{~g}}-\frac{d p}{d T}\right) \dot{T}=w_{\mathrm{f}} c_{p 1}\left(T_{\mathrm{f}}-T\right)-\lambda\left(w_{\mathrm{f}}-w_{\mathrm{c}}\right)-Q
$$

The vapor condensation or feed evaporation term is easily eliminated from the two equivalent temperature differential equations, giving the two-dimensional state 
description of the vapor phase alone. The state variables are the vapor phase temperature and the vapor phase volume,

$$
\begin{gathered}
V_{\mathrm{g}}\left(\rho_{\mathrm{g}} c_{p \mathrm{~g}}-\frac{d p}{d T}+\frac{\lambda \rho_{1}}{\rho_{1}-\rho_{\mathrm{g}}} \frac{d \rho_{\mathrm{g}}}{d T}\right) \dot{T}=w_{\mathrm{f}} c_{p 1}\left(T_{\mathrm{f}}-T\right)+\frac{\lambda \rho_{\mathrm{g}}}{\rho_{1}-\rho_{\mathrm{g}}}\left(w_{\mathrm{f}}-w_{\mathrm{p}}\right) \\
\dot{V}_{\mathrm{g}}=-\left(w_{\mathrm{c}}-w_{\mathrm{p}}\right) / \rho_{1}
\end{gathered}
$$

where the intermediate variable $w_{\mathrm{c}}$ is computed from eqn. (4). It should be noted that if the term $d p / d T$ is ignored, this reduces the time constant by about $14 \%$.

The energy balance still contains an unknown term which is the heat flux to the vapor phase surroundings. This flux is positive during increasing temperature and pressure transients. Since these are the most important cases, the analysis is limited to these. During such transients there will be no flashing from the liquid phase, only heat diffusion from the surface and into the liquid. The heat conductivity of the propane liquid (Engineering, Science Data Unit, Perry 1963) is found to be about $1 / 16$ of the heat conductivity of the rock, and the heat flux into the liquid phase during loading is ignored. Then the heat transfer to the rock wall $Q$ is described by the simple equation

$$
Q=\left(A_{\text {roof }}+A_{\text {wall }} V_{\mathrm{g}} / V_{\mathrm{o}}\right) U\left(T-T_{\mathrm{s}}\right)
$$

The rock temperatures throughout are described by a transient heat conduction equation in its traditional form (Carslaw and Jaeger 1959),

$$
\frac{\partial T}{\partial t}=a \nabla^{2} T ; \quad a=\left(\frac{k}{\rho c_{p}}\right)_{\text {rock }}
$$

Since the heat penetration into the rock is relatively small compared with the dimension of the vapor phase volume, the heat conduction into the rock is simplified to a one-dimensional heat diffusion.

$$
\frac{\partial T}{\partial t}=-a \frac{\partial^{2}}{\partial x^{2}} T
$$

This leads to a discretized set of equations for each layer of rock

$$
\left.\begin{array}{l}
\dot{T}_{i}=a^{*}\left(\Delta T_{i-1}-\Delta T_{i}\right) ; \quad \Delta T_{i}=T_{i}-T_{i+1} \\
a^{*}=k /\left(\rho c_{p} \Delta x^{2}\right)
\end{array}\right\}
$$

This means that the effective heat diffusion of the cavern corners and edges are ignored. The surface temperature of the rock and the boundary conditions for the difference equations (12) are

$$
\begin{aligned}
& T_{\mathrm{s}}=\left(\beta T+T_{1}\right) /(1+\beta) ; \beta=U \Delta x / k \\
& \Delta T_{\mathrm{o}}=2 \beta\left(T-T_{1}\right) /(1+\beta) ; \text { for } i=1 \\
& \Delta T_{\mathrm{n}}=0 \quad ; \text { for } i=n
\end{aligned}
$$

This is the complete model description of the single-phase vapor model. 


\section{A two-phase vapor-liquid model}

In a two-phase vapor-liquid model, the total liquid volume, and not only the surface of the condensate film, is assumed to be in equilibrium with the vapor phase. The energy balance is then taken over the complete cavern comprising both the vapor and the liquid phase. This model is not very likely, because it requires complete mixing of the liquid phase. Nevertheless, the results from this model are shown for comparison.

If the liquid is fed at the bottom of the liquid phase in the cavern, the material balance of the vapor phase is simply

$$
\frac{d}{d t}\left(\rho_{\mathbf{g}} V_{\mathrm{g}}\right)=w-w_{\mathrm{s}}-Q / \lambda
$$

where $w$ is the evaporation rate from the liquid interface. This variable is eliminated as before, assuming thermodynamic equilibrium. This yield a differential equation analogous to eqn. (7) but with a larger time constant

$$
\begin{aligned}
\left(V_{\mathrm{g}}\left(\frac{\lambda \rho_{1}}{\rho_{1}-\rho_{\mathrm{g}}} \frac{d \rho_{\mathrm{g}}}{d T}+\rho_{\mathrm{g}} c_{p \mathrm{~g}}-\frac{d p}{d T}\right)+V_{\mathrm{l}} \rho_{1} c_{p 1}\right) \dot{T}= & \frac{\lambda \rho_{1}}{\rho_{1}-\rho_{\mathrm{g}}}\left(w_{\mathrm{f}}-w_{p}\right) \\
& +w_{\mathrm{f}}\left(T_{\mathrm{f}}-T\right) c_{\mathrm{pl}}-\frac{\lambda \rho_{1}}{\rho_{1}-\rho_{\mathrm{g}}} w_{\mathrm{s}}-Q
\end{aligned}
$$

As can be seen, the difference between the two models is only in the time constant where the liquid volume heat capacity is now included. The right-hand sides of both eqn. (17) and eqn. (7) are equal. The ratio between the time constants of these two models varies quite a lot depending on the liquid loading. As an example take a $20 \%$ liquid loading. Then the time constant of eqn. (17) is in the order of three times that of eqn. (7), while the ratio between the two time constants at $80 \%$ liquid loading is about 34. In order to check which one of the two models would be most appropriate, the temperature transients should be tested out at various liquid loadings.

The dynamic state equation for the gas volume is similarly

$$
\dot{V}_{\mathrm{g}}=-\left(\left(w_{\mathrm{f}}-w+Q / \lambda\right)-w_{\mathrm{p}}\right) / \rho_{1}
$$

where the net evaporation is determined from

$$
\left(1-\rho_{\mathrm{l}} / \rho_{\mathrm{g}}\right)\left(w_{\mathrm{f}}-w+Q / \lambda\right)=w_{\mathrm{f}}-V_{\mathrm{g}} \frac{\partial \rho}{\partial T} \dot{T}-\left(\rho_{\mathrm{g}} / \rho_{1}\right) w_{\mathrm{p}}-w_{\mathrm{s}}
$$

The heat diffusion into the rock is computed in a similar way for both models, as it is assumed that the heat transfer from the liquid phase to the rock is much lower than from the vapor phase where the condensation is the dominating heat transfer mechanism.

\section{A simple illustration of pressure transients during unloading of an LPG carrier}

The consequences of a specified but fairly unlikely worst case of loading conditions into the cavern, may be simulated in order to check the risk of malfunctioning control equipment. If the control valve to the heat exchanger is left at its maximum opening and the feed flow rate is kept at its maximum value during the complete period of unloading, a typical transient in the temperature and pressure of the cavern, as 
shown in Fig. 2 would be the result. The failure of the temperature control loop is supposed to last unattended for about 6 hours, and then the feed to the cavern is shut off gradually during half an hour. If the single-phase model is used, the temperature increase of about $4.5^{\circ} \mathrm{C}$ is predicted, corresponding to about 0.8 bar pressure increase. This is close to the tolerable limit. This model also predicts a safety valve opening of about $20 \%$ maximum. Figure 2 also shows how the heat penetrates into the rock at 25 and $62.5 \mathrm{~cm}$ deep.
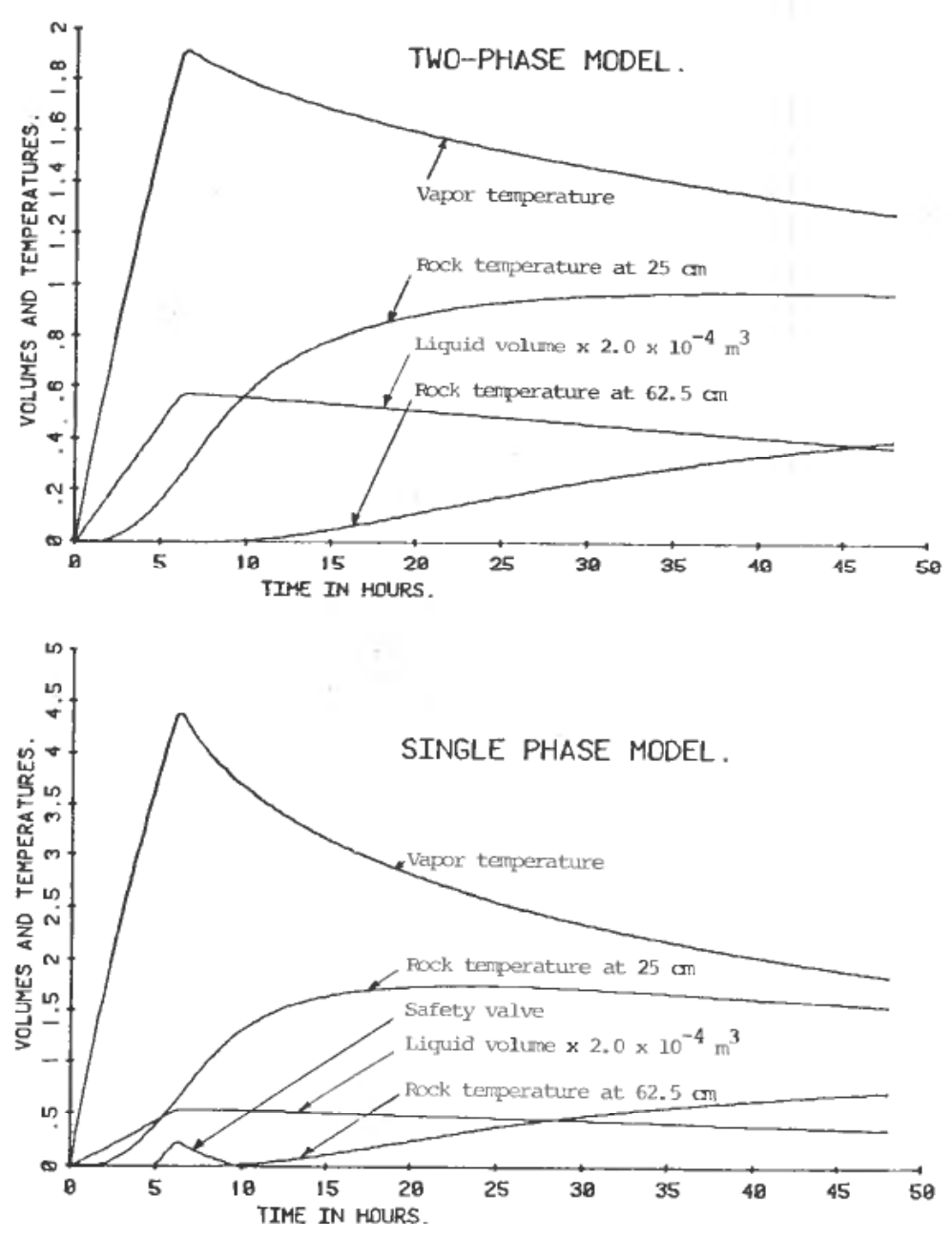

Figure 2. Simulated transients during a sudden rise in the feed enthalpy lasting 6 hour

The two-phase model predicts a substantially lower temperature increase, basically due to the thermal inertia of the liquid phase. The peak now appears somewhat less than $2^{\circ} \mathrm{C}$ (about $0.35 \mathrm{bar}$ ) and the safety valve remains closed. From the point of view of hazards caused by a control loop failure, it is of a great advantage to get the feed mixed into the liquid. This is particularly so at high liquid loading. On the other hand, the single phase model leads to the most conservative evaluation of the possible hazards, especially at the high liquid loadings of the cavern as shown. 


\section{A simple feed forward control strategy}

When the liquid propane storage has come to rest, the heat flux $Q$ and the flow rate through the safety valve are both zero. If the temperature after the preheater is properly adjused during the unloading of say an LPG carrier, it is clearly possible to make the disturbance on the cavern minimal by a feed temperature adjustment such that the right-hand side of the differential equations for the cavern temperature remains zero. This condition is independent of the choice of a single-phase or a twophase model, and gives a feed temperature equivalent to

$$
T_{\mathrm{f}}=T-\left(1-w_{\mathrm{p}} / w_{\mathrm{f}}\right) \lambda \rho_{\mathrm{g}} /\left(\left(c_{p}\right)_{1}\left(\rho_{1}-\rho_{\mathrm{g}}\right)\right)
$$

independent of the initial liquid loading of the cavern.

Normally the feed rate would be much larger than the take-off rate, and one might estimate the subcooling of the feed relative to the cavern temperature.

$$
T-T_{\mathrm{f}}=\Delta T=\lambda \rho_{\mathrm{g}} /\left(\left(c_{\mathrm{p}}\right)_{1}\left(\rho_{\mathrm{l}} \rho_{\mathrm{g}}\right)\right) \simeq 5 \cdot 1^{\circ} \mathrm{C}
$$

This control strategy would ensure a condensation of the vapor in the cavern just sufficient to remain a constant pressure when the liquid level rises during the feed of the cavern. The fairly cold feed would be in danger of forming hydrates in contact with water at the bottom. Both the hydrate precautions and the desired condensation effect certainly suggest the feed to the cavern to be introduced as a spray at the top of the vapor volume. The safety precautions against failing control equipment, however, favours the feed to the cavern to be introduced at the bottom in the liquid phase. This suggests an extra automatic feed shut-off system if the feed temperature goes above a certain upper safe limit.

\section{Estimation of most likely parameters for the heat and material balances from real} plant measurements

In real plant operation the feed control is not always perfect as suggested by the feed forward control law, both the feed rate as well as the feed temperature varies

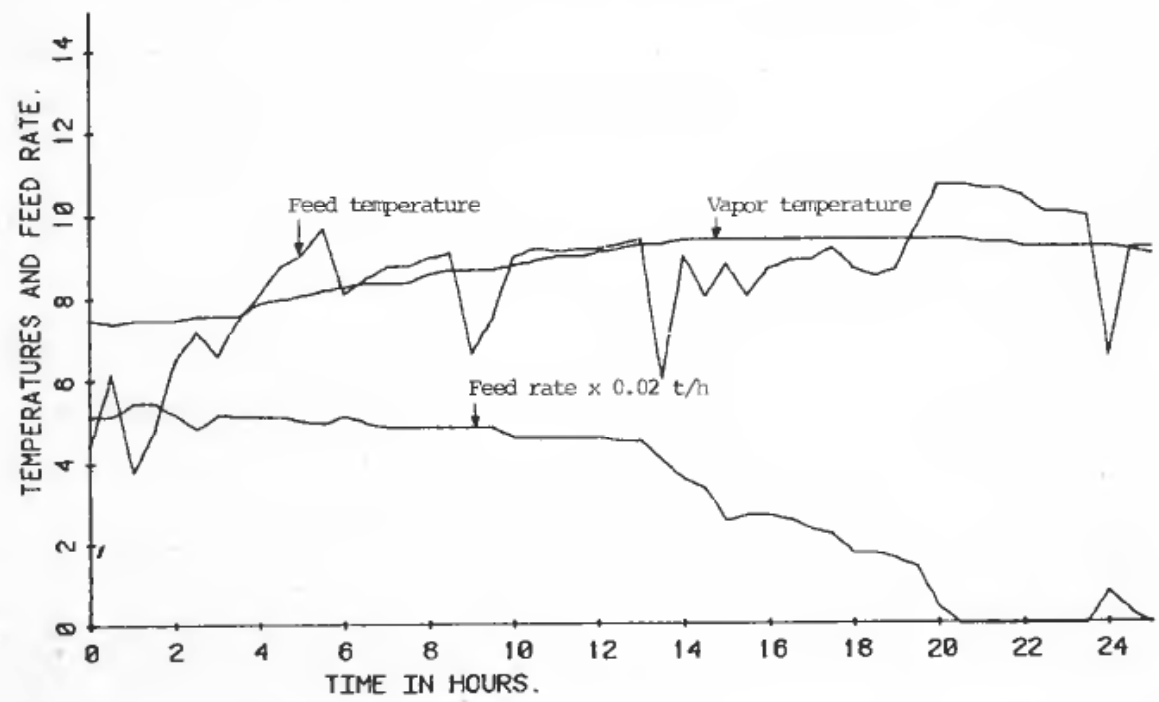

Figure 3. Real plant data for feed rate, feed temperature and vapor temperature in the cavern. 
substantially. This means that temperature transients will occur in the cavern, and these transients may be used for parameter estimation in the very simple models suggested. This was done during some of the first initial loadings of the cavern, where flow rate, feed temperature and cavern temperature in the vapor phase were recorded every half hour during a 24 hour unloading of an LPG carrier.

The typical history of liquid feed rate and temperature as well as the cavern response temperature are shown in Fig. 3. As is seen, the feed rate decreases as the carrier is unloaded, and the feed temperature is by no means constant. The cavern responds to a too high heat content of the feed by increasing the temperature in the vapor phase and consequently increasing the pressure in the cavern. The liquid level in the cavern was also measured and shows a typical integral of the flow rate as indicated in Fig. 4.

The time functions representing the feed rate and the feed temperature are now used as inputs to the mathematical models of the cavern. From this the response in temperature and liquid level is simulated. First, the two-phase model without any
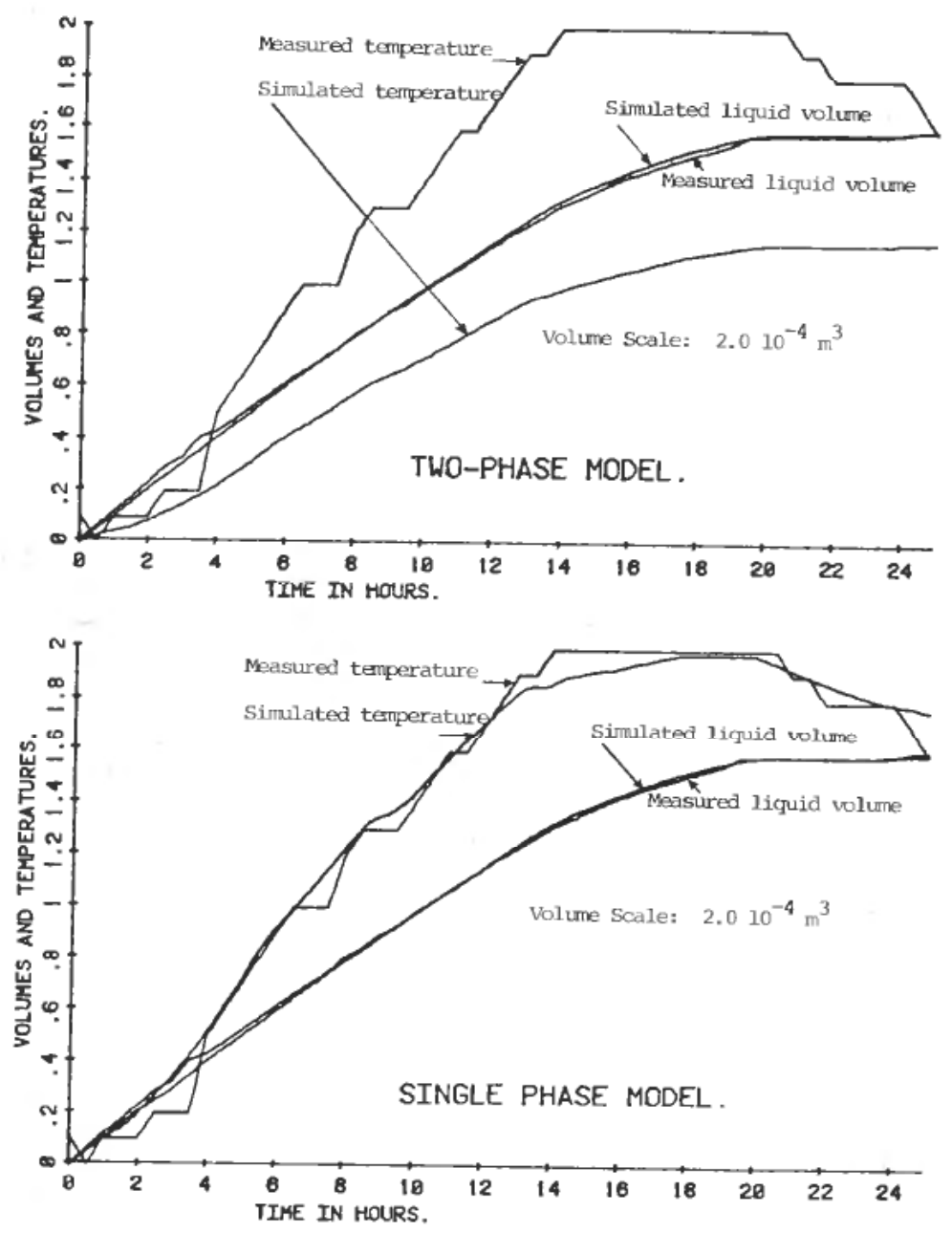

Figure 4. Comparison between the two models and real plant data. 
heat transfer to the surroundings is attempted. Secondly, the single-phase model with adjustable heat transfer and heat diffusion is applied.

As is shown for the two-phase model in Fig. 4, the material balance showing the simulated and measured liquid volume agrees quite well. The simulated temperature response, however, is very far from the real response in the cavern. The time constant in the two-phase model is too high, the cavern responds as if the liquid phase was not active as a thermal capacity. This suggests that the single-phase model as developed, would be a more appropriate model for the temperature response.

The standard deviation between the simulated and measured responses in temperature and liquid level were used as criteria for the fitting of the single-phase model. The three parameters were adjusted in a simple way manually. Those parameters were the heat transfer coefficient $U$, the rock heat conductivity $k$ and the height to width ratio of the cavern. The final results of the fitting are shown in Fig. 4, where the standard deviation of the liquid level response is in the order of less than $1 \%$, and of the temperature response is in the order of $3.5 \%$ or $0.07^{\circ} \mathrm{C}$.

The fit of the single-phase model is reasonably good considering that standard process instruments were used. Hence the order of magnitude of the heat transfer and the heat diffusion in the rock is established. These data may be used for later simulation (as the case was for the results presented in Fig. 2) or for later designs of similar caverns.

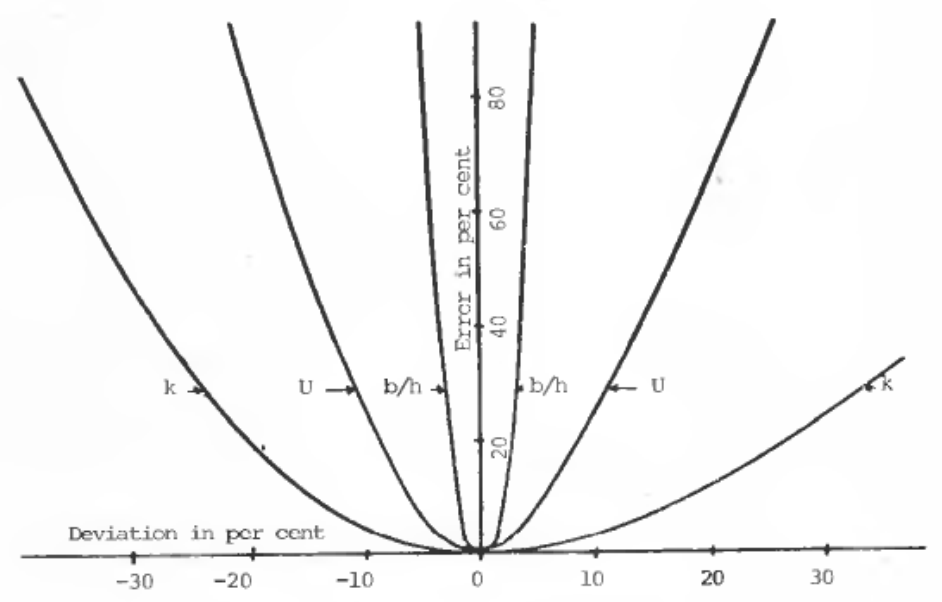

Figure 5. Parameter sensitivities.

The sensitivity of the mean square error function of the model fit to the parameters near the optimal fit is shown in Fig. 5. As is seen, the most sensitive parameter is the width to height ratio, but this parameter does not influence the temperature response, only the material balance on the liquid level response. The next two parameters are important for the temperature response, but there is practically no sensitivity in the liquid level response. In the temperature response the heat transfer coefficient is most significant, while the sensitivity to the rock heat conductivity is not very pronounced. The sensitivity in the residual to the parameters reflects in the accuracy by which the parameters may be estimated. 
Hence, the most likely values and the relative accuracy of the estimated parameters may be established as follows:

$\begin{array}{lllll}\text { Height to width ratio: } & 0.962 & & +5 \% & -5 \% \\ \text { Heat transfer coefficient: } & 145 & \mathrm{~W} / \mathrm{m}^{2} \mathrm{~K} & +27 \% & -22 \% \\ \text { Rock heat conductivity: } & 1.63 & \mathrm{~W} / \mathrm{m} \mathrm{K} & +87 \% & -43 \%\end{array}$

These data are all very likely and the residual of the model fit is very good, all conditions considered. One may therefore conclude that the single-phase model is an applicable one and that its parameter values are plausible.

\section{Possible improvement of the metering accuracy}

The simulated liquid level response may be used as a mean to check the accuracy of the liquid level instrument of the cavern. Owing to the condensation effect the liquid level will always show a systematic deviation from the integral of the flow. As long as the model applies properly as was the case here, the deviation between the simulated and measured level should be not more than $1 \%$ as a random error. For metering purposes, however, the accuracy required is certainly higher, and the material balance model has a fairly limited application in this respect. It may only be used as a checking device for the instruments.

It is interesting to notice, however, that there is a correlation between the response deviations in the temperature and the liquid level as shown in Fig. 6. In this figure the percentage deviations are plotted against time. The correlation coefficient between these two deviations is about $-0 \cdot 7$. This observation is what would be expected, that a low temperature follows from an higher degree of condensation and consequently a higher liquid level. The fluctuations in the degree of condensation as Fig. 6 indicates, is again an indication of large-scale turbulent fluctuations in the heat transfer and of the equilibrium conditions. This indeed limits the accuracy of the model and hence limits the incentive to further modelling of the transient behaviour of the cavern.

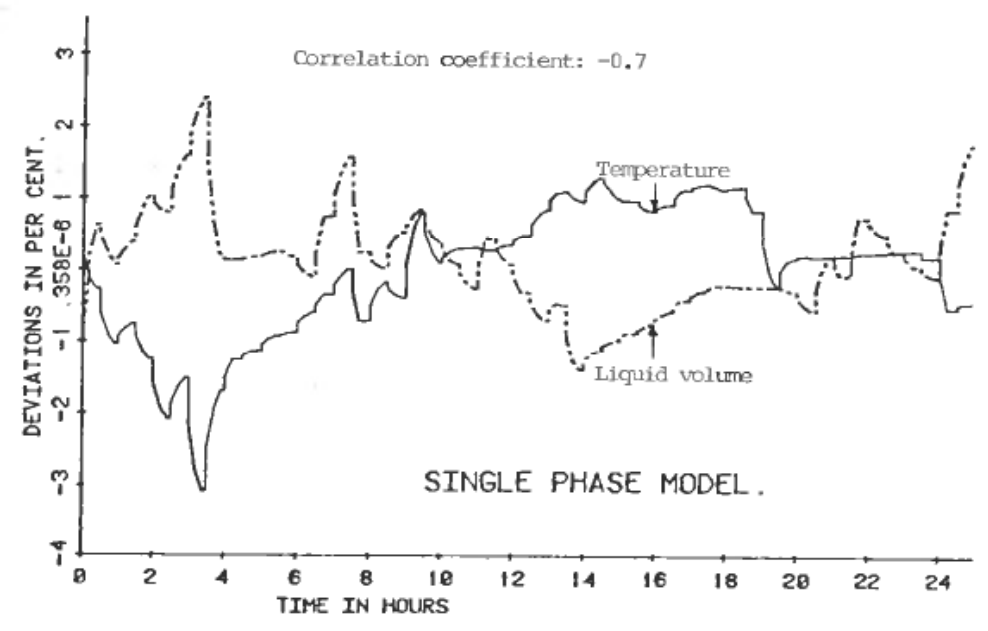

Figure 6. Correlation between model residuals. 


\section{Conclusion}

It may be concluded from the modelling, the simulation and the parameter estimation, that a reasonable description of transient loading of a closed cavern for LPG has been obtained. The heat transfer and the heat conduction parameters for the cavern, as estimated from real plant behaviour, are plausible and may be used for design and evaluation of future operations of new caverns.

The control of the temperature and pressure transients in a cavern is trivial, as the model as well as the real process responses indicate a very slow first-order process. The temperature of the feed should be kept constant at a level below the cavern temperature, which balances out the compression by condensation during loading of the cavern. In order to improve the safety of the loading operation, the feed should have independent and automatic trip systems, triggered by the integrated difference between the feed temperature and the cavern temperature. If this time integral violates a limit of safe operation, which could easily be obtained by simulation, the feed to the cavern should be shut off.

This analysis and the models developed are based on a single-component system, i.e. the vapor phase is pure propane vapor in equilibrium with the liquid. When this is the case, temperature and pressure are equivalent. If inert substances are present however, the pressure will be somewhat higher than the saturation pressure at the measured temperature, and this deviation may be used as an inert substance indicator. The order of magnitude of the heat transfer coefficient indicates small amount of inert substances, but not sufficient to have any real influence on the pressure. In principle, the estimated heat transfer coefficient may also be used as a fairly sensitive indicator of inert substances.

\section{REFERENCES}

Carslaw, H. S., and JAEger, J. C. (1959). Conduction of Heat in Solids (Oxford University Press).

Douglas, J. M. (1972). Process Dynamics and Control, Vol. 1, Analysis of Dynamic Systems (Englewood Cliffs: Prentice-Hall).

Engineering Sciences Data (1975). Item No. 75003 (London: Engineering Sciences Data Unit Ltd),

Harriot, P. (1964). Process Control (New York: McGraw-Hill).

LuYBen, W. L. (1973). Process Modelling, Simulation and Control for Chemical Engineers (New York: McGraw-Hill).

Perry, J. H. (editor) (1963). Chemical Engineers Handbook (New York: McGraw-Hill). 